\title{
PrEvention of posttraumatic contractuRes with Ketotifen 2 (PERK 2) - protocol for a multicenter randomized clinical trial
}

\author{
Ayoola Ademola ${ }^{1,2^{*}}$ D, Kevin A. Hildebrand ${ }^{1,3}$, Prism S. Schneider ${ }^{1,2,3}$, Nicholas G. H. Mohtadi ${ }^{1,3}$, Neil J. White ${ }^{3}$,
} Michael J. Bosse ${ }^{4}$, Alexandra Garven ${ }^{3}$, Richard E. A. Walker ${ }^{1,5}$ and Tolulope T. Sajobi ${ }^{2}$

\begin{abstract}
Background: Injuries and resulting stiffness around joints, especially the elbow, have huge psychological effects by reducing quality of life through interference with normal daily activities such as feeding, dressing, grooming, and reaching for objects. Over the last several years and through numerous research results, the myofibroblast-mast cellneuropeptide axis of fibrosis had been implicated in post-traumatic joint contractures. Pre-clinical models and a pilot randomized clinical trial (RCT) demonstrated the feasibility and safety of using Ketotifen Fumarate (KF), a mast cell stabilizer to prevent elbow joint contractures. This study aims to evaluate the efficacy of KF in reducing joint contracture severity in adult participants with operately treated elbow fractures and/or dislocations.

Methods/design: A Phase III randomized, controlled, double-blinded multicentre trial with 3 parallel groups (KF 2 $\mathrm{mg}$ or $5 \mathrm{mg}$ or lactose placebo twice daily orally for 6 weeks). The study population consist of adults who are at least 18 years old and within 7 days of injury. The types of injuries are distal humerus (AO/OTA type 13) and/or proximal ulna and/or proximal radius fractures (AO/OTA type $2 \mathrm{U} 1$ and/or 2R1) and/or elbow dislocations (open fractures with or without nerve injury may be included). A stratified randomization scheme by hospital site will be used to assign eligible participants to the groups in a 1:1:1 ratio. The primary outcome is change in elbow flexionextension range of motion (ROM) arc from baseline to 12 weeks post-randomization. The secondary outcomes are changes in ROM from baseline to 6, 24 \& 52 weeks, PROMs at 2, 6, 12, 24 \& 52 weeks and impact of KF on safety including serious adverse events and fracture healing. Descriptive analysis for all outcomes will be reported and ANCOVA be used to evaluate the efficacy KF over lactose placebo with respect to the improvement in ROM.
\end{abstract}

Discussion: The results of this study will provide evidence for the use of KF in reducing post-traumatic joint contractures and improving quality of life after joint injuries.

Trial registration: This study was prospectively registered (July 10, 2018) with ClinicalTrials.gov reference: NCT03582176.

Keywords: RCT, Ketotifen Fumarate, Contractures, DASH, OES, PCS, ROM

\footnotetext{
* Correspondence: ayoola.ademola@ucalgary.ca

${ }^{1}$ McCaig Institute of Bone and Joint, University of Calgary, Calgary, Canada

${ }^{2}$ Department of Community Health Sciences, University of Calgary, Calgary,

Canada

Full list of author information is available at the end of the article
}

(c) The Author(s). 2020 Open Access This article is distributed under the terms of the Creative Commons Attribution 4.0 International License (http://creativecommons.org/licenses/by/4.0/), which permits unrestricted use, distribution, and

reproduction in any medium, provided you give appropriate credit to the original author(s) and the source, provide a link to the Creative Commons license, and indicate if changes were made. The Creative Commons Public Domain Dedication waiver (http://creativecommons.org/publicdomain/zero/1.0/) applies to the data made available in this article, unless otherwise stated. 


\section{Background}

Joint contractures develop as a consequence of trauma, arthritis, or reconstructive procedures and can be functionally debilitating [1]. These injuries occur most commonly in the working age group (20-60 years), thus, representing a significant societal burden in North America [2-5]. Analysis of the Calgary Health Region database revealed that approximately 1200 elbow fractures or dislocations occurred in 2002-2005 [2-5]. These rates extrapolated to the Canadian population resulted in an estimated 20,000 elbow fractures or dislocations per year nationally. Assuming 1 in 8 will develop a joint contracture requiring operative intervention, an estimated $>2500$ operative procedures per year for elbow contracture in Canada [6-10]. Extrapolated to the US, this would yield over 25,000 operative procedures annually.

Over the last 20 years, our laboratory research has implicated a myofibroblast-mast cell-neuropeptide axis of fibrosis in post-traumatic joint contractures [11]. Ketotifen Fumarate (KF) is a medication that has anti-anaphylactic properties, due to the prevention of the synthesis and/or release of growth factors and mediators from mast cells. It has been used in the treatment of chronic asthma for over 40 years in humans. Post-market surveillance has confirmed the safety of KF (11, Bassler). Through a preclinical rabbit model of post-traumatic joint contractures, we have shown that KF, decreased contracture severity concomitant with decreased numbers of myofibroblasts, mast cells, neuropeptide containing nerve fibres, and measures of fibrosis in the joint capsule in a dose-dependent fashion [12-14]. It is the first and only agent demonstrating both a significant decrease in contracture severity in preclinical trials and a wide safety profile. A previously conducted pilot randomized clinical trial (NCT01902017) demonstrated safety of KF and coupled with preclinical animal studies informed the need to increase the sample size, use multiple doses to examine for a dose response, and further refine the trial population to more severe injuries requiring an operation in the Phase III randomized clinical trial (RCT).

The primary objective is to evaluate the efficacy of KF in reducing post-traumatic elbow joint contractures when compared to placebo in participants with elbow fractures or dislocations administered within 7 days of injury and for a duration of 6 weeks. There are two secondary objectives i.e. (1) Evaluate the efficacy of KF over lactose placebo with respect to Disability Arm Shoulder Hand (DASH), Oxford Elbow Score (OES), and Pain Catastrophizing Scale (PCS), (2) Evaluate the impact of KF on safety including serious adverse events, fracture healing and re-operation rates.

This is a Phase III randomized, controlled blinded multicentre efficacy trial with three parallel groups and a primary endpoint of elbow flexion-extension range of motion (ROM) arc at 12 weeks post-randomization (Fig. 1). Eligible participants will be randomized to receive KF 2 mg, KF 5 mg, or lactose placebo (PL) in a 1:1:1 allocation ratio. The medication is administered orally twice daily for 6 weeks.

\section{Methods/design \\ Participants, interventions, and outcomes}

Seventeen academic medical centres from Canada (fourteen) and the United States (three) will be participating in the trial (Appendix 1). The study population of interest are adults (defined as 18 years of age or older) with any combination of distal humerus, or proximal radius or ulna fractures, or elbow dislocations without pre-existing elbow arthritis or contractures (Table 1). A 7 day window from injury to starting medication is based on research indicating that the intervention is most effective if administered early in the healing process [15]. The exclusion criteria are broadly divided in those that predispose to elbow contracture (elbow specific), those that are consequences of using KF or lactose placebo (medication contraindications), and those that interfere with the ability follow the protocol (participant specific (Table 1)). Further, there is a lack of safety evidence for the use of KF during pregnancy or breast feeding.

\section{Interventions}

Eligible participants will be randomized in equal proportions to receiving KF $2 \mathrm{mg}$, KF $5 \mathrm{mg}$, or a PL. This trial follows from the feasibility PERK 1 RCT (Clinicaltrials.gov, NCT01902017) where a dose of $5 \mathrm{mg}$ was used, which is larger than the recommended dose of Ketotifen for the treatment of chronic asthma (1-2 mg twice daily) [11]. In choosing the second dose, the higher end of the recommended dose $(2 \mathrm{mg})$ was selected balancing the effectiveness to prevent contractures and decreasing the chance for side effects (sedation). The medications will be administered orally twice daily for a total of 6 weeks postrandomization. Ketotifen Fumarate is manufactured in 1mg capsules by TEVA Canada. Bay Area Research Logistics (BARL, Hamilton, Ontario, Canada) specializes in clinical trial medication packaging and distribution and will ensure blinding is achieved by over-encapsulation of each treatment, such that KF and PL capsules will appear identical. Ketotifen Fumarate is an antihistamine and sedation has been reported in $14 \%$ of people taking it in post-marketing surveillance [16]. Modifications to the allocated treatment will include discontinuing the capsules for serious suspected adverse reactions such as excessive drowsiness or skin rashes. Adherence to taking the medications will be stressed at the time of dispensing the capsules and reinforced through weekly calls by research personnel, as well as daily diarizing by participant. Adherence assessments with pill counts will be completed at the 2- and 6-week follow-up visits. A relatively short six-week medication administration will facilitate adherence. The only restricted medication that may not be taken simultaneously with KF is antihistamines. If these are required in the first 6 weeks 
Elbow Fractures or Dislocations

Fig. 1 PERK 2 Trial Design

after randomization, the trial medication will be discontinued. There are no rescue medications for KF.

\section{Assignment of interventions}

Screening, randomization and enrollment is organized through the Epidemiology Coordinating and Research
Centre (EPICORE) at the University of Alberta. A stratified block randomization scheme by hospital site will be used to assign eligible participants at baseline to KF 2 $\mathrm{mg}$, KF $5 \mathrm{mg}$, or PL in a 1:1:1 ratio using a computergenerated randomization scheme (i.e. conducted on REDCap over the internet via a desktop computer or a

Table 1 Eligibility Criteria

\begin{tabular}{|c|c|}
\hline INCLUSION CRITERIA & EXCLUSION CRITERIA \\
\hline $\begin{array}{l}\text { - Adults ( } \geq 18 \text { years or older); skeletally mature } \\
\text { - Injury to the elbow with any of the following: } \\
\text { o Distal } 1 / 3 \text { humerus fracture (AO/OTA Type } 13 \text { ) } \\
\text { o Proximal } 1 / 3 \text { ulna fracture } \\
\text { (AO/OTA Type } 2 \text { U1) } \\
\text { O Proximal } 1 / 3 \text { radius fracture } \\
\text { (AO/OTA Type } 2 \text { R1) } \\
\text { o Elbow dislocation } \\
\text { - Open fractures with/without nerve injury can be } \\
\text { included } \\
\text { - Participant presents within } 7 \text { days or less between } \\
\text { injury and trial recruitment } \\
\text { - Participant requires operative treatment of the } \\
\text { fracture } \\
\text { - Participant has a negative urine pregnancy test }\end{array}$ & $\begin{array}{l}\text { - Elbow specific } \\
\text { o Pre-existing elbow contracture, osteoarthritis, inflammatory arthritis, gout or non-specific } \\
\text { monoarticular arthritis of the injured elbow } \\
\text { o Inability to mobilize elbow injury within } 21 \text { days of injury or surgery } \\
\text { o Total Elbow for fracture treatment } \\
\text { o Prior Elbow injury or surgery } \\
\text { o Bilateral elbow fractures and / or dislocations } \\
\text { - Medication contraindications } \\
\text { o Oral hypoglycemic medications } \\
\text { o History of epilepsy } \\
\text { o Lactose intolerance } \\
\text { o Any female who is pregnant or nursing } \\
\text { o Severe hepatic impairment } \\
\text { o Severe renal impairment } \\
\text { o Male or female of reproductive age unwilling to use } 2 \text { methods of contraception } \\
\text { - Participant specific } \\
\text { o Unable to maintain follow-up (no fixed address, plans to move out of town in the next year, } \\
\text { states unable to comply with protocol, etc...) } \\
\text { o Has cognitive impairment or language difficulties that would impede the reliable } \\
\text { completion of questionnaires } \\
\text { o Concomitant musculoskeletal or visceral injuries preventing elbow physiotherapy } \\
\text { o Unwilling or Unable to provide written informed consent. }\end{array}$ \\
\hline
\end{tabular}


web-enabled smart phone) in randomly assigned block sizes of 3,6 or 9. All participants, care providers, research personnel, investigators, outcome assessors and data entry personnel will be blinded to intervention groups. Emergency unblinding will be permitted.

\section{Outcomes}

The primary outcome is the range of motion (ROM) in the flexion-extension arc and forearm pronation-supination arc obtained with a hand-held goniometer. Loss of ROM is a major reason for patients to consult physicians following elbow injury. Secondary outcomes include patient reported outcomes measures (PROMs) such as the Disability of the Arm, Shoulder, and Hand questionnaire (DASH), Pain Catastrophizing Scale (PCS) and Oxford Elbow Score (OES). The upper extremity scoring scale DASH is a validated 30-item PROM tool for disorders of the elbow with a range from 0 (least disability) to 100 (most disability). The PCS is a 13-item PROM with aggregates into three subscale scores assessing rumination, magnification, and helplessness that are combined into an overall score that range between 0 (no pain) and 42 (catastrophizing pain) [17-20]. PCS has been used in the assessment of injuries to the elbow, wrist, and hand where results were dependent on these behaviors. The OES is a 12-item valid measure of the outcome of elbow surgery in English and Dutch languages [21, 22]. There are three domains which include elbow function, pain, and social-psychological with values ranging from 0 (greatest severity) to 100 (least severity). The OES has been used in trauma populations [2326]. It was reported that the OES performed well in assessing elbow surgical outcomes while the DASH was not responsive enough to warrant its exclusive use as an outcome of elbow surgery [27]. The other secondary outcomes include safety (adverse events (AE) and serious adverse events (SAE)), re-operations and fracture healing. For $\mathrm{AE}, \mathrm{SAE}$ and re-operation, we are interested in the description and number of occurrence, while for fracture healing, our interest is the number of participants with disappearance of radiographic fracture lines overtime.

\section{Data collection}

Potential participants will be identified when scheduled for an operation for their injured elbow through the emergency department, hospital admission, or at an outpatient clinic. The surgeons will be involved in introducing the participants to the trial, discuss the potential concern regarding elbow contracture and the importance of preventing this disabling complication. Surgeons will also obtain permission from the participant for research personnel to contact the participant. Eligible participants who sign informed consent will be allocated to one of the three treatment arms and begin taking the medication the same day (Table 2). Participant assessments include standardized phone calls at 1, 3, 4 and 5 weeks post-randomization to review medication use, ask about adverse events and confirm clinic appointments. Clinic visits, where ROM, PROMs, X-rays, and survey for adverse events are completed, occur at 2, 6, 12, 24 and 52 weeks postrandomization. X-rays will be gathered and stored at the Calgary Image Processing and Analysis Centre (4 views, including obliques). The presence or absence of visible fracture line to indicate healing and location of heterotopic ossification will be adjudicated by 3 independent reviewers. Pill counts will be performed at the 2- and 6-week followup visits (Table 2).

\section{Sample size calculation}

The sample size was estimated based on analysis of covariance model (ANCOVA) with respect to the mean change from baseline (as represented by the injured elbow at 2week follow up visits) to flexion-extension arc of motion at week 12, on the basis of a two-sided test at the $0.05 \alpha$ level. A total of 702 participants $(\approx 234$ participants per arm) is needed to achieve about $95 \%$ power to detect a minimum improvement of 10 degrees among the treatment arms, assuming a standard deviation of 19.81, and an overall dropout rate of $11 \%$. This sample size will have about $99.1 \%$ statistical power to detect a MCID in mean change (i.e. 10) in DASH scores [28] across treatment groups between baseline and week 12. Furthermore, the total sample size of 702 participants will ensure that we have at least $98 \%$ power to detect a minimum of $20 \%$ difference in the proportion of participants achieving functional flexion-extension arc of motion between placebo and treatment arms where a functional elbow ROM is defined as at least $30^{\circ}-130^{\circ}$ flexion-extension arc for activities of daily living [29]. Seventeen centres will provide participants for the trial. The 13 sites outside of Calgary are forecasted to enroll 30 particpants each and the $4 \mathrm{Cal}$ gary centres are forecast to enroll the remaining 312 participants (Appendix 1).

\section{Statistics analysis}

Descriptive analysis will be used to summarize ROM measurements, PROMs, safety (AE and SAE), re-operation and radiographic fracture healing. The analysis will follow the intention- to-treat protocol. Analysis of covariance (ANCOVA) will be used to model the impact of KF on ROM (change in 2 and 12 weeks of the injured elbow) adjusting for sex, age, injury classification and site. Similar analysis will be used to assess the impact of KF on in DASH, PCS and OES at 12 weeks, adjusting for these covariates. Mixed-effects regression analysis (effect of time) will be used to examine the impact of KF on changes in ROM and PROMs, adjusting for the earlier covariates. Logistic regression will be used to examine the impact of KF on radiographic fracture line disappearance (i.e. healing) 
Table 2 Schedule of enrolment, interventions, and assessments

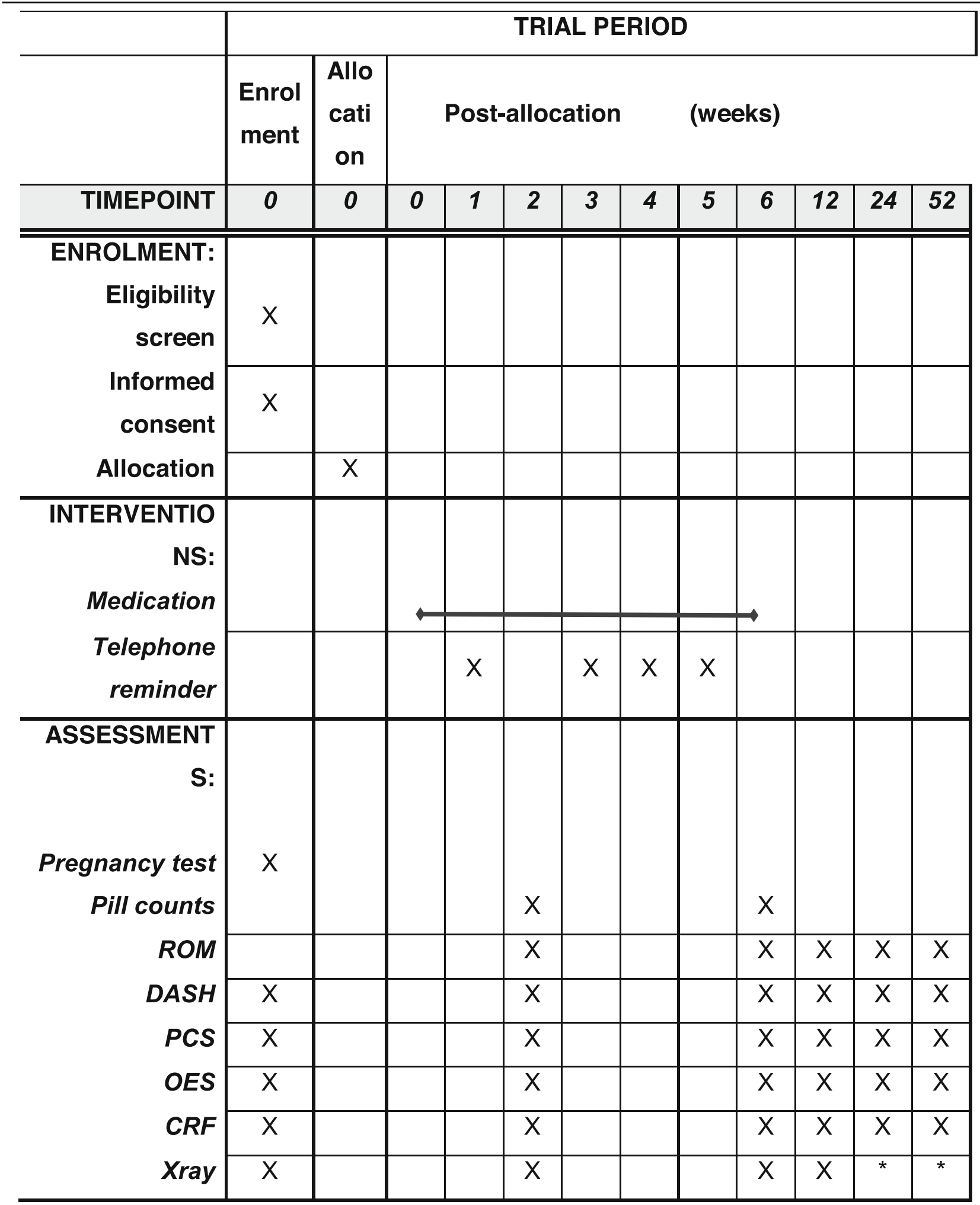

${ }^{*}$ If indicated for delayed or nonunion 
adjusting for sex, age, NSAID, smoking status. Sensitivity analysis will be used to evaluate the robustness of study findings to different methods for handling missing data [30]. Specifically, we will compare study conclusions based on complete data analysis, available data analysis, and use of multiple imputation based on Monte Carlo Markov Chain approach that adjusts for site, age andsex. Exploratory subgroup analyses to confirm treatment effect using different covariates such as range of motion (ROM), sex, age, side of injury is the same as dominant hand, race, concurrent injury and PROM i.e. DASH and OES.

\section{Data monitoring}

The Data Monitoring Committee (DMC), which will be formed, consist of an orthopaedic surgeon, clinical trialist and biostatistician but not involved with the proposed clinical trial in any manner and will be independent of the sponsor. Data will be reviewed twice yearly by the DMC via teleconference including recruitment and follow-up visit rates, SAEs, and descriptive statistics of the baseline demographics. Reports from the DMC will go to the Trial Steering Committee (TSC). The planned interim analysis will be shared with the DMC and a meeting arranged via teleconference for discussion and recommendations with the TSC. An interim analysis will be conducted when $50 \%$ of the participants have completed the primary outcome measure (ROM at the week 12 assessment). The DMC will evaluate whether there is an increased risk of KF use compared with PL. The parameters determined a priori to discontinue the trial include:

1. Greater than two times fatal or life-threatening SAEs that are Definitely or Probably related to KF (use Suspected Unexpected Serious Adverse Reaction SUSAR).

2. Greater than three times difference in the DASH and OES between groups.

3. Greater than three times the number of participants with non-functional ROM (30 degrees to 130 degrees flexion-extension arc) between groups.

If any one of the above criteria are met, the DMC will recommend to the TSC that the trial will be terminated prior to completion. The Trial Steering Committee (TSC) will include the Principal Investigator, Trial Biostastician, Project Manager, Orthopaedic Clinical Trialist, and three site Prinicipal Investigators or Project Managers. The TSC will meet quarterly to review trial performance and adjust the RCT as required based on safety and performance inputs. All outcome measures will be evaluated at the completion of the trial when the last participant has reached 52 weeks post-randomization.

\section{Participant care}

Concomitant care covers operations, medications, and rehabilitation/splints. Together, the participant and surgeon will determine the type of operation. This management will not be randomly assigned, but determined by injury characteristics. The operation can be performed before or after randomization; however randomization and first dose administration must occur within 7 days of injury. Analgesia may be provided by acetaminophen, opioids and/ or NSAID as required. Antihistamines use will be excluded for the 6 weeks that participants are taking trial medication since KF is an antihistamine. If possible, a local physiotherapist will direct a standardized home therapy program for all participants consisting of active ROM exercises performed three times per day with 20 repetitions for elbow flexion-extension arc and forearm pronation-supination arc. Progress will be monitored and adherence to home therapy will be reinforced every 2 weeks during the clinic visitation to the local physiotherapist. Stretching splints are instituted once it is clear that physiotherapy alone is insufficient, which occurs a minimum of 12 weeks postrandomization.

\section{Safety}

For the purposes of this trial, AEs will be defined as any unfavourable medical occurrence that a participant experiences, including sign, symptom or disease that occurs during participation in the trial whether or not considered trial medication related [7, 8, 28, 31-36]. Serious Adverse Events will be defined as any untoward medical occurrence that: results in death or is lifethreatening; requires inpatient hospitalization or prolongation of existing hospitalization; leads to persistent or significant disability and/or incapacity; causes a congenital anomaly and/or birth defect. All AEs and SAEs will be collected as a secondary outcome of the trial in a standardized format on the case report forms (CRFs). Suspected Adverse Reaction (SAR) is a type of AE where there is a reasonable possibility that the trial medication $\mathrm{KF}$ caused the AE. For all AEs, a temporal relationship will be observed in relation to the KF administration. Ketotifen Fumarate is administered over 6 weeks, and a 1-week washout period will be included. Thus, to be considered a SAR, the AE would have to occur within 7 weeks of randomization. The "definitely related" and "probably related" designations would indicate the $\mathrm{AE}$ is a SAR. For pregnancy, any conception that arises within the 6 weeks and/or within a 3-month interval after the 6-week course of KF will be followed to ascertain whether any birth defects, congenital anomalies or loss of pregnancy occurred. Unblinding will occur in order to ascertain what the participant was randomized in order 
to provide counselling on what the fetus may have been exposed to KF or PL [31-35, 37-40]. All AEs or SAEs will be classified as expected or unexpected. Expected AEs are defined in the product monograph (PM) for KF and include recognized complications of an elbow fracture population undergoing an operation for the injury [36]. Unexpected AEs or SAEs are all other AEs or SAEs not defined as expected. Suspected unexpected serious adverse reactions (SUSAR) are defined as unexpected SAEs where there is causal relation between the SAE and use of KF.

\section{Discussion}

Currently, if contractures develop then only solution is an operation followed by 6-9 months of rehabilitation. An oral medication used during the recovery from the original injury to prevent post-traumatic contracutres is a simpler and safer approach. Contractures complicate other orthopaedic conditions or procedures and positive results in post-trauamtic contractures in elbow may warrant study of the use of KF in preventing contractures in other orthopaedic conditions or procedures. Therefore, we believed that the PrEvention of Posttraumatic Joint contractuRes With Ketotifen 2 (PERK 2) trial will provide evidence for a treatment (Ketotifen) to prevent contractures following joint injuries. If an improvement in ROM is observed, the treatment will have a significant impact on quality of life. A multi-centre design involving seventeen sites is planned to achieve a recruitment rate of 234 participants per year over 3 years to obtain a total of 702 participants. The multi-dosage regimen will provide an opportunity to determine the optimal dosage for treatment. The safety of KF for a new indication will be assessed, including radiographic fracture healing.

\section{Supplementary information}

Supplementary information accompanies this paper at https://doi.org/10. 1186/s12891-020-3139-2.

Additional file 1. Trial Sites.

\section{Acknowledgements}

Not applicable.

\begin{abstract}
Authors' contributions
KAH:- Principal Investigator, Fund acquisition, study design, drafting and reviewing of manuscript. AA:- Draft, revision and submission of manuscript. PSS:- Study design and reviewing of manuscript, NM:- Review of manuscript, NJW:- Review of manuscript, MJB:- Review of manuscript, AG:- Review of manuscript, RAW:- Review of manuscript \& TTS:- Review of manuscript. All authors have read and approved the final manuscript.
\end{abstract}

\section{Funding}

This grant for this trial was obtained from United States Department of Defense (Funding Reference Number: OR160026). According to the definition of funding, this is an externally funded, however, the funding body does not have any other role in this trial aside for the funding that was provided.

\section{Availability of data and materials}

All data generated or analysed during this study are included in this published article [and its supplementary information files].

\section{Ethics approval and consent to participate}

The ethics approvals for the trial were obtained from the Conjoint Health Research Ethics Board (CHREB) at the University of Calgary (REB 17-0609) and Institutional Review Board (IRB)/ Research Ethics Board (HSREB) of other participation sites. All Protocol modifications will be reported to IRBs/REBs, regulatory bodies and communicated to participating trial sites. In addition, we will obtain signed written consent from all participants before randomization in the study. On trial completion, the data will be migrated off the clinical trial data management platform and housed in research data archival service for a period appropriate to satisfy the regulatory and ethical requirements of the trial.

\section{Consent for publication}

Not applicable. The manuscript does not have any patient information or details.

\section{Competing interests}

There is no completing interest in the trial, however, the funding for the trial was obtained from the US Department of Defense. The funding body does not have any other role in this trial.

\section{Author details}

${ }^{1}$ McCaig Institute of Bone and Joint, University of Calgary, Calgary, Canada. ${ }^{2}$ Department of Community Health Sciences, University of Calgary, Calgary, Canada. ${ }^{3}$ Department of Surgery, University of Calgary, Calgary, Canada. ${ }^{4}$ Department of Orthopaedic Surgery, Carolinas Medical Center, Charlotte, USA. ${ }^{5}$ Department of Radiology, University of Calgary, Calgary, Canada.

Received: 16 October 2019 Accepted: 13 February 2020

Published online: 24 February 2020

\section{References}

1. Fergusson D, Hutton B, Drodge A. The Epidemiology of Major Joint Contractures: A Systematic Review of the Literature. Clin Orthop Relat Res. 2007:456:22-9. https://doi.org/10.1007/s00198-005-0011-z.

2. Sheps DM, Kemp KA, Hildebrand KA. Population-based incidence of proximal radial and ulnar fractures among adults in a Canadian metropolitan area. Curr Orthop Pract. 2012;23(4):364-8.

3. Sheps DM, Kemp KAR, Hildebrand KA. Population-based incidence of distal humerus fractures among adults in a Canadian urban center. Curr Orthop Pract. 2011;22(5):437-42.

4. Sheps DM, Kemp KAR, Hildebrand KA. Elbow dislocations in a Canadian metropolitan health region: a 3-year population-based incidence study. Shoulder Elbow. 2010;2:281-6. https://doi.org/10.1111/j.1758-5740.2010. 00084.x.

5. Sheps DM. The incidence of elbow injuries in the city of Calgary. The incidence of elbow injuries in the city of Calgary. University of Calgary; 2006. from: https://dspace.ucalgary.ca/bitstream/1880/44849/1/Sheps_ MSc_2006_Med.pdf

6. Myden C, Hildebrand K. Elbow joint contracture after traumatic injury. J Shoulder Elb Surg. 2011;20:39-44.

7. Doornberg JN, van Duijn PJ, Linzel D, Ring DC, Zurakowski D, Marti RK, Kloen P. Surgical treatment of intra-articular fractures of the distal part of the humerus. Functional outcome after twelve to thirty years. J Bone Joint Surg. 2007;89A:1524-32.

8. Forthman C, Henket M, Ring DC. Elbow dislocation with intra-articular fracture: the results of operative treatment without repair of the medial collateral ligament. J Hand Surg. 2007;32A:1200-9.

9. Lindenhovius ALC, Jupiter JB. The Posttraumatic Stiff Elbow: A Review of the Literature. J Hand Surg. 2007;32A(10):1605-23 from: http://www. sciencedirect.com/science/article/B6WJK-4RB1666-V/2/be065cb2eef3d780d3 b3a2680ffb5828.

10. Cooney WP III, Morrey BF. Contractures of the elbow. In: The Elbow and its Disorders. Philadelphia: W.B. Saunders Company; 1993. p. 464-75. from: http://www.sciencedirect.com/science/article/B6WB8-45WHRYN-3/2/361 dbdbb1f64f9a48eb2e1857a5f4d99. 
11. Monument MJ, Hart DA, Salo PT, Befus AD, Hildebrand KA. Neuroinflammatory mechanisms of connective tissue fibrosis: targeting neurogenic and mast cell contributions. Adv Wound Care. 2015;4(3):137-51.

12. Monument MJ, Hart DA, Befus AD, Salo PT, Zhang M, Hildebrand KA. The mast cell stabilizer ketotifen reduces joint capsule fibrosis in a rabbit model of post-traumatic joint contractures. Inflamm Res. 2012;61:285-92.

13. Monument MJ, Hart DA, Befus AD, Salo PT, Zhang M, Hildebrand KA. The mast cell stabilizer ketotifen fumarate lessens contracture severity and myofibroblast hyperplasia: a study of a rabbit model of posttraumatic joint contractures. J Bone Joint Surg. 2010;92A:1468-77.

14. Schneider PS, Johal H, Zhang M, Hart D, Befus A, Salo P, Fan CY, Liang X, Hildebrand $K$. The dose response effect of ketotifen fumarate on posttraumatic joint contractures. Transactions of the orthopaedic research society; 2016. p. 0221.

15. Gallant-Behm CL, Reno C, Tsao H, Hart DA. Genetic involvement in skin wound healing and scarring in domestic pigs: assessment of molecular expression patterns in (Yorkshire $\times$ red Duroc) $\times$ Yorkshire backcross animals. J Investig Dermatol. 2007;127(1):233-44.

16. Maclay WP, Crowder D, Spiro S, Turner P. Postmarketing surveillance: practical experience with ketotifen. Br Med J (Clin Res Ed). 1984;288(6421): 911-4.

17. Sullivan MJ. PCS The Pain Catastrophizing Scale User Manual 2009. PCS the pain Catastrophizing scale user manual 2009. Montreal; 2009. http://sullivanpainresearch.mcgill.ca/pdf/pcs/PCSManual_English.pdf.

18. Teunis JT, et al. Catastrophic Thinking Is Associated With Finger Stiffness After Distal Radius Fracture Surgery. J Orthop Trauma. 2015;29(10):e414e420.

19. Vranceanu AM, Bachoura A, Weening A, Vrahas M, Smith RM, Ring D. Psychological factors predict disability and pain intensity after skeletal trauma. J Bone Joint Surg Am. United States. 2014;96(3):e20.

20. Golkari S, Teunis T, Ring D, Vranceanu AM. Changes in depression, health anxiety, and pain Catastrophizing between enrollment and 1 month after a radius fracture. Psychosomatics. 2015;56(6):652-7.

21. Dawson J, Doll H, Boller I, Fitzpatrick R, Little C, Rees J, Jenkinson C, Carr AJ. The development and validation of a patient-reported questionnaire to assess outcomes of elbow surgery. J Bone Joint Surg Br. England. 2008; 90(4):466-73.

22. De Haan J, Goei H, Schep NW, Tuinebreijer WE, Patka P, den Hartog D. The reliability, validity and responsiveness of the Dutch version of the Oxford elbow score. J Orthop Surg Res. England. 2011;6:39.

23. Dawson J, Doll H, Boller I, Fitzpatrick R, Little C, Rees J, Carr A. Comparative responsiveness and minimal change for the Oxford elbow score following surgery. Qual Life Res. Netherlands. 2008;17(10):1257-67.

24. Iordens GIT, Van Lieshout EMM, Schep NWL, de Haan J, Tuinebreijer WE, Eygendaal D, Van Beeck E, Patka P, Verhofstad MH, Den Hartog D, FuncSiE Trial Investigators. Early mobilisation versus plaster immobilisation of simple elbow dislocations: results of the FuncSiE multicentre randomised clinical trial. Br J Sports Med. 2015. https://doi.org/10.1136/bjsports-2015-094704.

25. Aitken SA, Jenkins PJ, Rymaszewski L. Revisiting the 'bag of bones'. Bone Joint J. 2015;97-B(8):1132-8.

26. Fawi H, Lewis J, Rao P, Parfitt D, Mohanty K, Ghandour A. Distal third humeri fractures treated using the Synthes ${ }^{\mathrm{TM}} 3.5-\mathrm{mm}$ extra-articular distal humeral locking compression plate: clinical, radiographic and patient outcome scores. Shoulder Elbow. 2015;7(2):104-9.

27. Dawson J, Doll H, Boller I, Fitzpatrick R, Little C, Rees J, Carr A. Specificity and responsiveness of patient-reported and clinician-rated outcome measures in the context of elbow surgery, comparing patients with and without rheumatoid arthritis. Orthop Traumatol Surg Res. 2012;98(6):652-8.

28. Beaton DE, Katz JN, Fossel AH, Wright JG, Tarasuk V, Bombardier C. Measuring the whole or the parts? Validity, reliability and responsiveness of the Disabilities of the Arm, Shoulder and Hand outcome measure in different regions of the upper extremity. J Hand Ther. 2001;14:128-46 from: http://www.ejbjs.org/cgi/content/abstract/84/12/2168.

29. Morrey BF, Askew LJ, Chao EY. A biomechanical study of normal functional elbow motion. J Bone Joint Surg. 1981;63A(6):872-7.

30. Toutenburg H, Rubin DB. Multiple imputation for nonresponse in surveys. Stat Papers. 1990;31(1):180.

31. Grahnén A, Lönnebo A, Beck O, Eckernäs S-Å, Dahlström B, Lindström B. Pharmacokinetics of ketotifen after oral administration to healthy male subjects. Biopharm Drug Dispos. 1992 May;13(4):255-62.
32. Food and Drug Administration Center for Drug Evaluation and Research (2018). Guidance for Industry: Pregnant Women: Scientific and Ethical Considerations for Inclusion in Clinical Trials (draft).

33. Food and Drug Administration Center for Drug Evaluation and Research (2009). Guidance for clinical investigators, Sponsors, and IRBs: AE Reporting to IRBs - Improving Human Subject Protection.

34. Food and Drug Administration Center for Drug Evaluation and Research (2013). Guidance for industry and investigators: safety reporting requirements for IND, BA/BE Studies.

35. Food and Drug Administration Center for Drug Evaluation and Research (2015). Guidance for Industry: Safety Assessment for IND Safety Reporting (draft).

36. Teva Canada Limited. (2012). Product Monograph. Zaditen (Ketotifen Fumarate). http://tevacanada.com/ImageAndPdf.ashx?Objectld=e7007c3a-32 fe-46bb-990c-e55dd3b8826e\&DataType= ProductMonographPDF\&ObjectMethod=Value\&Language = English.

37. ICH-E2A. (1995). Guideline for industry. Clinical Safety Data Management: Definitions and Standards for Expedited Reporting.

38. ICH Harmonised Tripartite Guideline: Guideline for Good Clinical Practice. J Postgrad Med. 2001;47(3):199.

39. ICH-E6. (1996). Guidance for Industry. Good Clinical Practice: Consolidated Guidance. U.S. Department of Health and Human Services, Food and Drug Administration, Centre for Drug Evaluation and Research (CDER), Centre for Biologics Evaluation and Research (CBER).

40. Canadian Association of Research Ethics Boards. Guidance on Reporting of Unexpected Problems including Adverse Events to Research Ethics Boards in Canada. 2010

\section{Publisher's Note}

Springer Nature remains neutral with regard to jurisdictional claims in published maps and institutional affiliations.
Ready to submit your research? Choose BMC and benefit from:

- fast, convenient online submission

- thorough peer review by experienced researchers in your field

- rapid publication on acceptance

- support for research data, including large and complex data types

- gold Open Access which fosters wider collaboration and increased citations

- maximum visibility for your research: over $100 \mathrm{M}$ website views per year

At $\mathrm{BMC}$, research is always in progress.

Learn more biomedcentral.com/submissions 